\title{
A New Sampling Frequency Selection Scheme in Undersampling Systems
}

\author{
Yoshio KUNISAWA $^{\dagger \text { a) }}$, Naohiro SAHARA ${ }^{\dagger \dagger}$, Hiroshi SHIRAI ${ }^{\dagger \dagger}$, and Hisato IWAI ${ }^{\dagger \dagger \dagger \dagger}$, Members
}

\begin{abstract}
SUMMARY In software defined radio systems, placing the analog-todigital converter (ADC) near the antenna part in the block diagram of the receiver is desired to improve the flexibility of the system. The radio frequency (RF) sampling method, in which the received signal is sampled at the RF stage, realizes such structure. The undersampling is a potential method to sample the RF signal using the existing consumer ADCs because high speed ADCs are required in the traditional methods, such as Nyquist sampling or the oversampling of the RF signal. This paper presents a technique to determine the minimum sampling frequency to undersample the separated multiple wireless systems simultaneously. In addition, this paper proposes a frequency selecting scheme that enables selection of a lower sampling frequency by receiving at least the desired transmission channels in the wireless system signals. This paper also provides a result of performance analysis of the proposed scheme.

key words: software defined radio, undersampling, sampling frequency, $R F$ sampling
\end{abstract}

\section{Introduction}

As technology advances for personal mobile communications, making use of multiple communication services is desired at mobile terminals. Software Defined Radio (SDR) technology is the focus of much attention to realize such terminals [1], [2]. In SDR based terminals, the signal processing that is usually executed by hardware such as an ASIC (Application Specific IC) or a custom IC, is processed by software using DSP, FPGA or other reconfigurable processors. To extend the digital processing area and make the terminal applicable to many communication systems, it is desirable to set the ADC nearer the antenna part in receiver block diagrams. In a usual baseband sampling receiver, some analog devices such as an oscillator, mixer or filter restrict the flexibility of the receiver, because the parameters or the values targeting a specific system are fixed and can not be changed in such devices. To make a receiver more flexible, it is desirable to sample and digitize the received radio frequency signal at the RF frequency. The RF sampling scheme expands the region of the digital signal processing, and makes the receiver more programmable. How-

\footnotetext{
Manuscript received March 4, 2005.

Manuscript revised May 31, 2005.

${ }^{\dagger}$ The author is with KDDI R\&D Laboratories Inc., Kamifukuokashi, 356-8502 Japan.

${ }^{\dagger}$ The author is with KDDI Corporation, Tokyo, 102-8460 Japan.

${ }^{\dagger \dagger}$ The author is with Chuo University, Tokyo, 112-8551 Japan.

${ }^{+1 \dagger}$ The author is with Doshisha University, Kyotanabe-shi, 6100394 Japan.

a)E-mail: kuni@kddilabs.jp

DOI: 10.1093/ietcom/e88-b.11.4170
}

ever, a high speed ADC is required for the RF sampling receiver because the ADC needs to sample the high frequency signal and convert it to a digital signal. As one of the solutions to this, we consider the undersampling scheme, in which the frequency conversion and the sampling are performed at the same time using a sampling frequency less than the Nyquist frequency of the sampled signal [3]-[5]. In Ref. [3], the computation method for a sampling frequency of RF undersampling was proposed to sample and receive the signals of multiple communication systems simultaneously using single ADC. The paper presents the conditions to avoid the influences of interferences from the aliasing signal of the other systems. Using the proposed schemes, each channel of multiple communication systems can be received by one ADC. But the sampling frequency becomes higher when receiving the signal of a communication system that occupies wide bandwidth.

In the beginning part of this paper, the computation method described in Ref. [3] is shown as the original. And, a new modified frequency selection scheme is proposed to solve the problem mentioned above. The interference to a desired transmitting channel is avoided by the proposed scheme. The scheme offers a sampling frequency selection method according to the bandwidth of a transmitting channel, not heavily dependent on the system bandwidths. As a result, the sampling frequency becomes lower. The effect of the decrease in sampling frequency is evaluated by quantitative analysis.

\section{Undersampling [3]-[5]}

\subsection{Undersampling}

Undersampling is a sampling technique that utilizes the aliasing signal caused by using a sampling frequency lower than the Nyquist frequency. And signal is sampled and converted to low frequency simultaneously. Figure 1 shows an example of a block diagram of an RF sampling receiver. The signal received at the antenna is amplified by the LNA (Low Noise Amplifier). Thereafter, only the de-

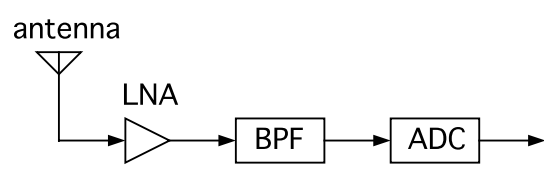

Fig. 1 Block diagram of RF sampling receiver. 
sired signal is passed by the BPF (Band Pass Filter). Using appropriate sampling frequency $F_{S}$, the desired signal is frequency-converted to a frequency range between 0 and $F_{S} / 2$. Out-band interference and noises not rejected by the $\mathrm{BPF}$ are also frequency-converted to the same frequency range. Thus, the signal can be frequency-converted without an oscillator or a mixer by undersampling. Here, the relation between the desired signal's center frequency $F_{C}$ and the frequency-converted signal's center frequency $F_{I F}$ is indicated by Eq. (1).

$$
\text { If } f i x\left(\frac{F_{C}}{\frac{F_{S}}{2}}\right) \text { is }\left\{\begin{array}{l}
\text { even, } \quad F_{I F}=\operatorname{rem}\left(F_{C}, F_{S}\right) \\
\text { odd, } \quad F_{I F}=F_{S}-\operatorname{rem}\left(F_{C}, F_{S}\right)
\end{array}\right.
$$

In Eq. (1) $f i x(a)$ is a function to obtain the value after omitting the decimal places of the value $a$, and $\operatorname{rem}(a, b)$ is a function to obtain the remainder of the value a divided by the value $b$. The center frequency of the signal after frequencyconversion by undersampling is obtained by Eq. (1). An appropriate sampling frequency by which all the bandwidth of the desired wireless system is converted to a frequency range between 0 and $F_{S} / 2$, should be selected to avoid interference from the aliasing signal of the desired system. Such sampling frequency $F_{S}$ and the frequency-converted signal's center frequency $F_{I F}$ are required to satisfy both Eq. (2) and Eq. (3).

$$
\begin{aligned}
& 0<F_{I F}-\frac{B W}{2} \\
& F_{I F}+\frac{B W}{2}<\frac{F_{S}}{2}
\end{aligned}
$$

In Eqs. (2) and (3), $B W$ indicates the bandwidth of the desired system. The undersampling scheme is applicable when the sampling frequency $F_{S}$ satisfying Eqs. (2) and (3) exists.

\subsection{Undersampling for Multiple System Signals}

To sample the signals of multiple systems simultaneously by single ADC, the signals are required to be pre-bandpass filtered, and the frequency bandwidths of the signals are required to be restricted. As an example, the structure of a receiver in which the signals of the multiple systems are sampled simultaneously is shown in Fig. 2, as well as the conceptual view of the spectrum.

First, the center frequency of each system after the frequency-conversion is calculated by Eq. (1). Then the sampling frequency, by which the signals of each system avoid interferences from the system itself, is selected by finding the value satisfying Eqs. (2) and (3). A condition should be added to sample the signals of multiple systems simultaneously, and the sampling frequency is selected. Equation (4) is the condition to sample the signals of two systems without mutual interference.

$$
\left|F_{I F_{1}}-F_{I F_{2}}\right| \geq \frac{B W_{1}+B W_{2}}{2}
$$

The subscript in Eq. (4) is the number identifying the

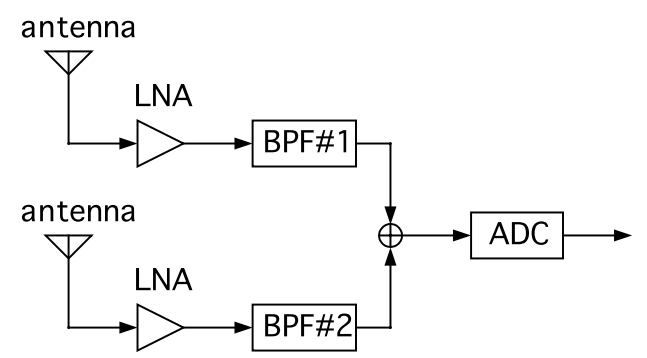

(a) Reciever structure for undersampling multiple system signals.

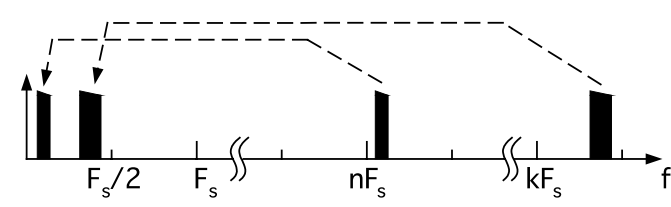

(b) Conceptual view of spectrum.

Fig. 2 Undersampling for multiple system signals.

two systems. For example, $F_{I F 1}$ indicates the frequencyconverted center frequency of System 1. Similarly, $B W_{1}$ indicates the bandwidth of System 1. Equation (4) can be expanded to be applicable for $N$ systems. Equation (5) is the condition to sample the signals of $N$ systems by single ADC simultaneously.

$$
\left|F_{I F_{b}}-F_{I F_{a}}\right| \geq \frac{B W_{b}+B W_{a}}{2} \quad\left\{\begin{array}{l}
a=2, \cdots, N \\
b=1, \cdots, a-1
\end{array}\right.
$$

The subscripts a and b in Eq. (5) identify the systems' number.

By applying Eqs.(1)-(3) and (5), the sampling frequency that can be used to sample the signals of $N$ systems is selected.

\section{Proposed Scheme}

In the previous section, a sampling frequency selection scheme to sample the signals of multiple systems simultaneously was shown. The signals of entire multiple systems can be sampled using such a scheme without receiving the influence of the aliasing image. However, sampling the signals using the sampling frequency over twice the sum of all systems' bandwidths is required to satisfy Nyquist's sampling theorem. Therefore, the scheme has a problem in that the sampling frequency becomes high when the bandwidth of any receiving system is wide or many systems are desired to be received. In this paper, we propose a new sampling frequency selection scheme by which lower sampling frequency can be selected with the aim of receiving only the desired channels of each system.

\subsection{Sampling Frequency Selection Scheme to Receive Signal of Desired Channel}

$F_{I F}$ and $F_{I F c h}$, the center frequencies of a system and a desired channel after frequency-conversion respectively, are settled by Eqs. (1) and (6). 


$$
\text { If fix }\left(\frac{F_{c h}}{\frac{F_{S}}{2}}\right) \text { is }\left\{\begin{array}{l}
\text { even, } F_{I F_{c h}}=\operatorname{rem}\left(F_{c h}, F_{S}\right) \\
\text { odd, } F_{I F_{c h}}=F_{S}-\operatorname{rem}\left(F_{c h}, F_{S}\right)
\end{array}\right.
$$

In Eq. (6), $F_{c h}$ indicates the center frequency of the desired channel before frequency-conversion. The center frequency of a desired channel is revealed by Eq. (6). In order to receive and demodulate the signal of the desired channel, it is required that the frequency-converted signal of the desired channel is not influenced from any aliasing signals. Therefore, it is necessary to select a sampling frequency satisfying Eqs. (7) and (8).

$$
\begin{aligned}
& F_{I F_{c h}}-\frac{B W_{c h}}{2}>-\left(F_{I F}-\frac{B W}{2}\right) \\
& \frac{F_{S}}{2}-\left(F_{I F_{c h}}+\frac{B W_{c h}}{2}\right)>\left(F_{I F}+\frac{B W}{2}\right)-\frac{F_{S}}{2}
\end{aligned}
$$

In Eqs. (7) and (8), $B W_{c h}$ indicates the bandwidth of the desired channel. Using the sampling frequency satisfying Eqs. (7) and (8), the signal of the desired channel is received without the influence of the aliasing image.

\subsection{Sampling Frequency Selection Scheme for Multiple Systems}

In addition to the constraint of the sampling frequency described in Sect. 3.1, it is necessary to select a sampling frequency by which the frequency-converted signals of each desired channel are not influenced by the aliasing signals of other systems, to receive and demodulate every signal of each desired channel. Here is an example of receiving each signal of two channels in two systems. Firstly, select the sampling frequency by which each system and each desired channel satisfy Eqs. (1) and (6)-(8). Then pick up the sampling frequency by which the signals of each desired channel are not influenced by the signals of the aliasing images of other systems, using Eqs. (9) and (10).

$$
\begin{aligned}
& \left|F_{I F_{c h_{2}}}-F_{I F_{1}}\right| \geq \frac{B W_{c h_{2}}+B W_{1}}{2} \\
& \left|F_{I F_{2}}-F_{I F_{c h_{1}}}\right| \geq \frac{B W_{2}+B W_{c h_{1}}}{2}
\end{aligned}
$$

By the sampling frequency satisfying both Eqs. (9) and (10), the signals of the desired channels in each system can be undersampled without receiving the influence of the signals caused from another system.

Once the frequency of the desired channel is decided, the sampling frequency can be selected by the abovementioned process. When the frequency of the desired channel is changed, the sampling frequency is selected again using the same process. It is usual that the clock signal is generated by SG (Signal Generator) such as PLL (Phase Locked Loop) or NCO (Numerical Controlled Oscillator) and is supplied to the ADC in the actual receivers. The process does take some moment, but, because the clock signal is usually digitally generated using the $\mathrm{NCO}$, it takes little time to change the sampling frequency in the proposed scheme.
Equations (9) and (10) can be generalized to Eq. (11). By Eq. (11), the sampling frequency to receive the signals of $N$ desired channels in $N$ pairs of wireless systems, can be decided.

$$
\begin{aligned}
& \left|F_{I F_{b}}-F_{I F_{c h_{a}}}\right| \\
& \geq \frac{B W_{b}+B W_{c h_{a}}}{2} \quad\left\{\begin{array}{l}
a=1, \cdots, N \\
b=1, \cdots, N \\
a \neq b
\end{array}\right.
\end{aligned}
$$

\section{Quantitative Analysis}

In this section, the frequency selection schemes for receiving entire systems' signals and the desired channels' signals in the systems by undersampling, are compared. And the effect for decreasing the sampling frequency is evaluated quantitatively.

\subsection{System Model}

The system model used to evaluate the sampling frequency selection scheme is shown in Fig. 3. After the signals received at each antenna are amplified by LNA, the outband signal is suppressed by BPF. The bandwidth restricted signals are mixed with each other and undersampled by an ADC simultaneously. Then the signals of the desired channels are demodulated by each demodulation processing. Here, the characteristics in the case of receiving two systems' signals are assumed and evaluated. Table 1 shows the parameters of the two systems used in the evaluation. System 1 with a center frequency of $2122.5 \mathrm{MHz}$ and System 2 with a center frequency of $1906.55 \mathrm{MHz}$ are used. System 1 has eleven channels over the frequency of $2116.25 \mathrm{MHz}$ with $1.25 \mathrm{MHz}$ channelization. System 2 has 87 channels over the frequency of $1893.65 \mathrm{MHz}$ with $0.3 \mathrm{MHz}$ channelization.

Note that in proposed scheme, the sampling frequency should be variable to sample the signals of any combination

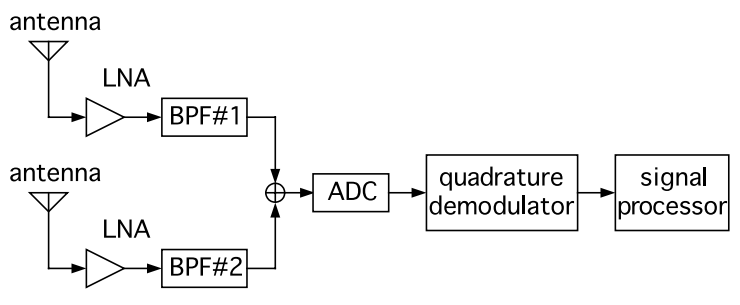

Fig. 3 System model

Table 1 Parameters of systems.

\begin{tabular}{|c|c|c|}
\hline & System 1 & System 2 \\
\hline $\begin{array}{c}\text { Carrier Frequency } \\
\mathrm{F}_{\mathrm{C}} \text { [MHz] }\end{array}$ & 2122.5 & 1906.55 \\
\hline $\begin{array}{c}\text { System Bandwidth } \\
\text { BW [MHz] }\end{array}$ & 13.75 & 26.1 \\
\hline $\begin{array}{c}\text { Channel Bandwidth } \\
\text { BW }_{\text {ch }}[\mathrm{MHz}]\end{array}$ & 1.25 & 0.3 \\
\hline
\end{tabular}


of channels in each systems.

4.2 Sampling Frequency to Sample Signals of Two Desired Systems (Conventional Scheme)

Figure 4(a) shows the relation between the sampling frequency and the frequency of the desired systems after frequency-conversion in the case of receiving entire systems' signals. In Fig. 4(a), the horizontal axis indicates the sampling frequency, and the vertical axis indicates the frequency after frequency-conversion. For example, by undersampling the signal using the sampling frequency of 83.0 MHz, System 1 is converted to the frequency range between $28.6 \mathrm{MHz}$ and $42.4 \mathrm{MHz}$, and System 2 is converted to the frequency range between $-10.7 \mathrm{MHz}$ and $15.5 \mathrm{MHz}$. If the frequency after frequency-conversion exceeds the upper constraint $\left(F_{S} / 2\right)$ or is below the lower constraint $(0)$, the aliasing signal of the system itself arises. So in the case of the sampling frequency of $83.0 \mathrm{MHz}$, a part of the signal of System 1 beyond $41.5 \mathrm{MHz}$ turns back. And as a result, at the frequency over $40.6 \mathrm{MHz}(=41.5-(42.4-41.5))$, the signal receives influence from the signal of the system itself. Similarly, a part of the signal of System 2 below $0 \mathrm{MHz}$ turns back. At the frequency below $10.7 \mathrm{MHz}$ $(=0-(-10.7))$ the signal receives influence from the signal

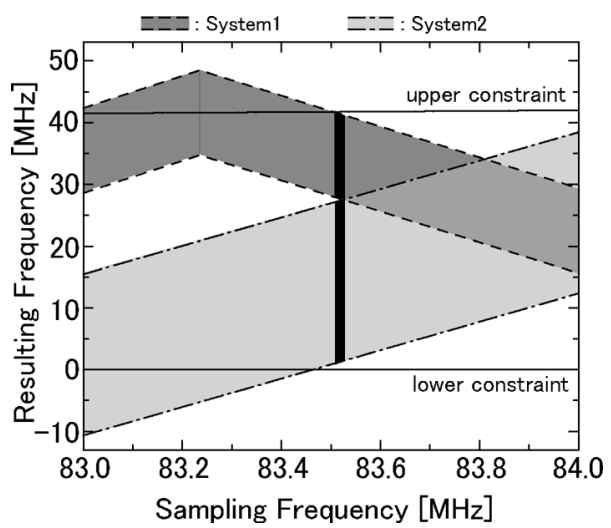

(a) Conventional.

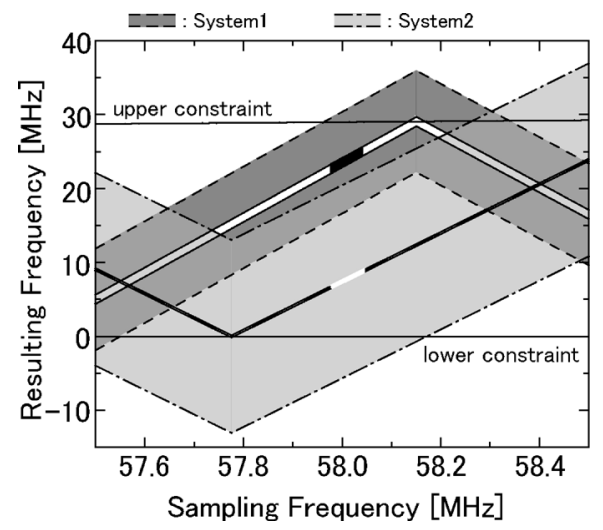

(b) Proposed.

Fig. 4 Relation between sampling frequency and frequency after frequency-conversion. (Near minimum sampling frequency) of the self system.

Hence, it is required that the frequency-converted signals of each system exist in the frequency range between the upper constraint and the lower constraint and they don't overlap each another, to receive two entire systems' signals without the influence of the aliasing signals of the self-system or another system. In Fig. 4(a), the regions of the sampling frequency and the frequency after frequencyconverting the systems, satisfying the conditions described above, are indicated in black. The minimum sampling frequency for undersampling is around $83.5 \mathrm{MHz}$ from Fig. 4(a).

\subsection{Sampling Frequency to Sample Signals of Desired} Channels in Two Systems (Proposed Scheme)

Figure 4(b) shows the relation between the sampling frequency and the frequency of desired systems after frequency-conversion in the case of receiving the signals of the desired channels in two systems. Here, two desired channels are assumed the center channels of the systems, and are indicated by solid lines in Fig. 4(b).

The signals of the desired channels are required not to overlap with the signals of the self-system or the other system in the frequency domain. Figure 4(b) indicates the minimum sampling frequency satisfying this condition is around $58.0 \mathrm{MHz}$.

Figure 5 shows the conceptual view of sampling frequency reduction. It is necessary to select the sampling frequency $F_{S}$ no to generate overlap over all frequency bands of each system in the conventional scheme. Every signal of received systems shall be frequency-converted to a frequency between 0 to $F_{S} / 2$ without the overlap. Therefore, $F_{S} / 2$ should be a larger value than the sum of each systems' bandwidth. In contrast, it does not matter in the proposed scheme if signals other than the desired channels receive the interference. Therefore, $F_{S} / 2$ can be decreased less than the total of all the received system bandwidths. In addition, the number of sampling frequencies available for undersampling can be increased using the proposed scheme.

When the number of the received systems increases and the total bandwidth of the received systems is wide, the minimum sampling frequency becomes higher and the number of the sampling frequencies is decreased. The bandwidth of the frequency-converted signal of each system can be regarded as narrower than the actual system bandwidth allowing the overlap of the other signals than the desired channels in the proposed scheme. Therefore the proposed scheme improves the possibility that the minimum sampling frequency can be decreased and the number of the available sampling frequencies can be increased also when the number of received systems increases.

\subsection{Reduction of Sampling Frequency}

Figure 6 shows the relation between the sampling frequency and the frequency of System 1 and System 2 af- 


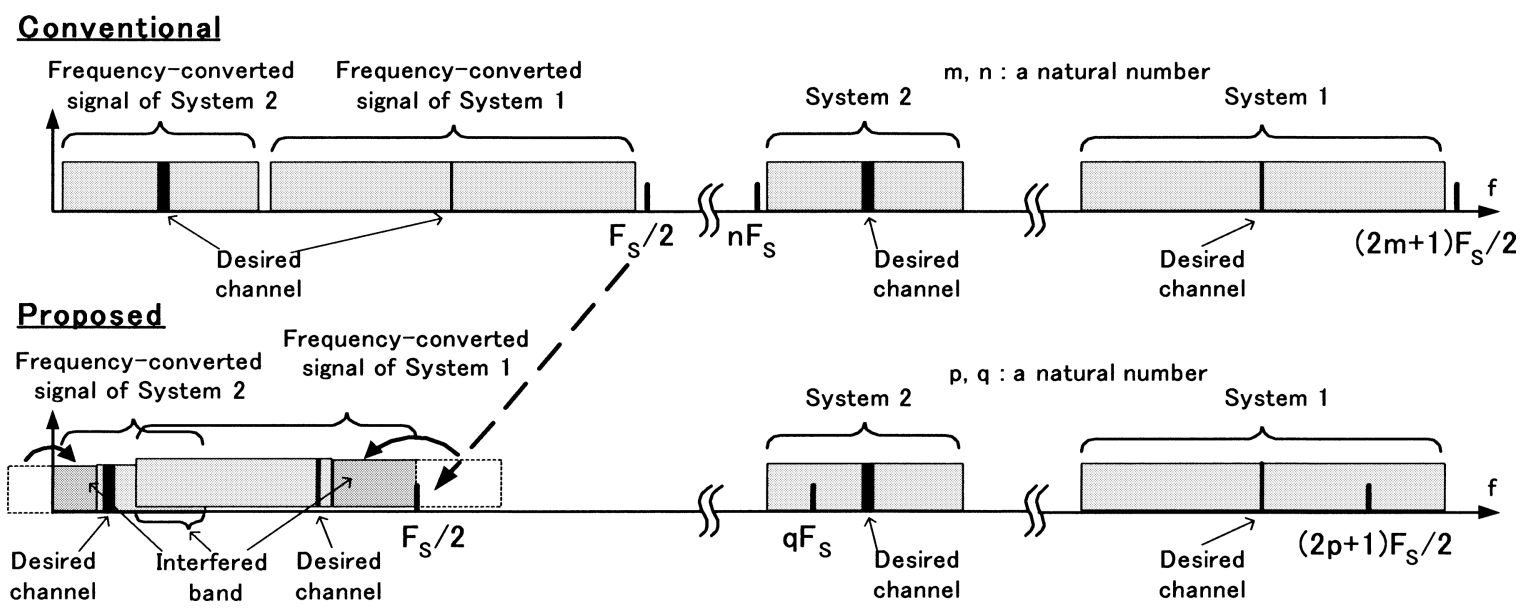

Fig. 5 Conceptual view of sampling frequency reduction.

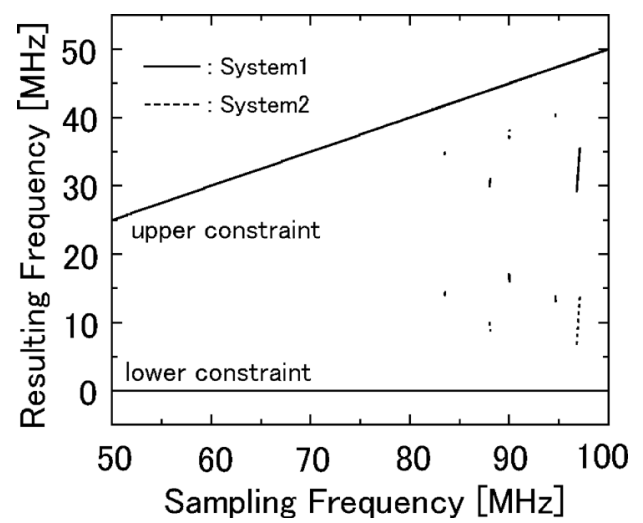

(a) Conventional.

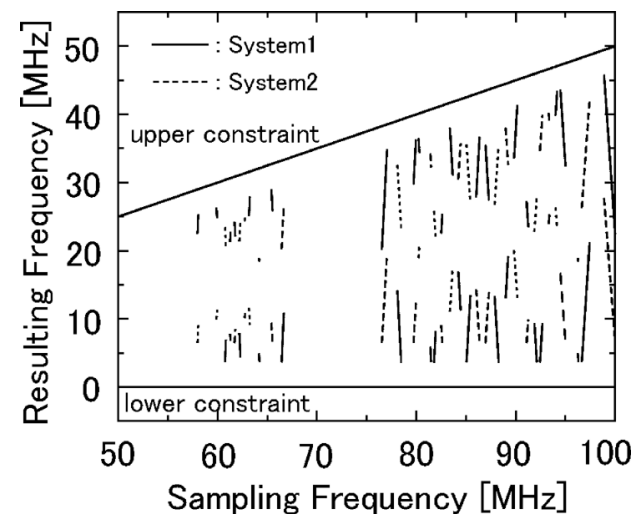

(b) Proposed.

Fig. 6 Relation between sampling frequency and frequency after frequency-conversion. (Sampling frequency between $100 \mathrm{MHz}$ from $50 \mathrm{MHz})$

ter frequency-conversion. Figure 6(a) shows the center frequency of two desired systems in the case of receiving the entire systems' signals. And Fig. 6(b) shows the center frequency of two desired channels in the case of receiving the signals of the desired channels in two systems. For example, Fig. 6(a) indicates only around $83.5 \mathrm{MHz}$ may be used for the sampling frequency of undersampling in the frequency range from $80.0 \mathrm{MHz}$ to $85.0 \mathrm{MHz}$, and the center frequen-

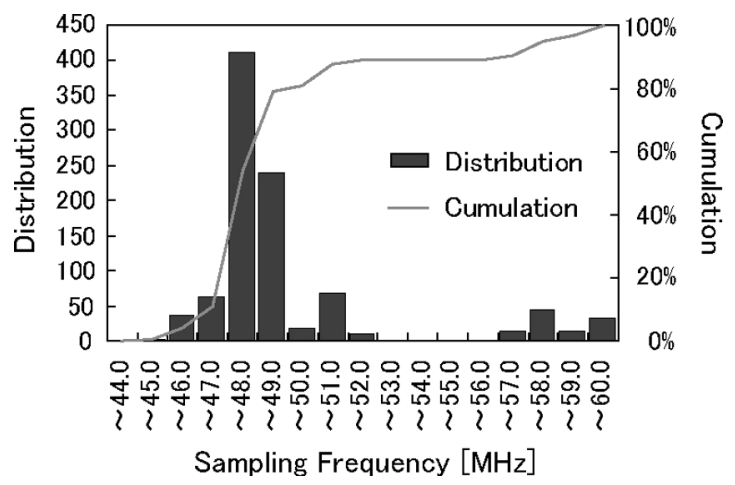

Fig. 7 Distribution of sampling frequency.

cies of System 1 and 2 are frequency-converted to $35.0 \mathrm{MHz}$ and $15.0 \mathrm{MHz}$, respectively.

The proposed scheme decreases the minimum sampling frequency as described in the previous section. The reason is that the sampling frequency to sample the entire signals of two systems is beyond twice the sum of the bandwidth of each system (Eq. (12)); however, the sampling frequency to sample the signals of each desired channel in each system is beyond the sum of the bandwidth of both systems and both desired channels (Eq. (13)).

$$
\begin{aligned}
& F_{S} \geq 2 \times\left(B W_{1}+B W_{2}\right) \\
& F_{S} \geq B W_{1}+B W_{2}+B W_{c h_{1}}+B W_{c h_{2}}
\end{aligned}
$$

By comparing Fig. 6(a) and Fig. 6(b), the proposed scheme can increase the number of the sampling frequency available for undersampling between $80 \mathrm{MHz}$ and $100 \mathrm{MHz}$.

The results presented in Figs. 4(b) and 6(b) are obtained assuming the desired channels are the center channels of the systems. Figure 7 shows the minimum sampling frequency distribution of all combinations $(11 \times 87$ sets $)$ of channels in both systems. Using the proposed scheme, the signals can be sampled by the sampling frequency under $50 \mathrm{MHz}$ in over $80 \%$ of combinations of channels in the systems. The average sampling frequency of all combinations of channels is around $49 \mathrm{MHz}$. As a result, the proposed 
scheme can decrease the sampling frequency effectively.

The requirements for ADCs can be eased by a lower sampling frequency realized by the proposed scheme. In addition, the signal processing performance and the processing speed required for the digital signal processing can be suppressed by smaller amount of the sampled data. It can be expected that the simultaneous usage of multiple broadband wireless communications should be required in the future ubiquitous communication systems. In such cases, there exists possibility that the sampling frequency becomes very high in the conventional scheme. The proposed scheme is very much effective in such cases, and is a very important technique to realize the next generation SDR terminals.

\section{Conclusion}

This paper describes a new sampling frequency selection scheme to undersample the signals of multiple systems in different frequency bands, simultaneously. While the scheme requires variable-rate compatible ADCs circuits, it effectively decreases the sampling frequency. Consequently power consumption at the digital signal processing can be reduced by the lower sampling frequency. This scheme is particularly effective in future broadband wireless systems where the system bandwidth becomes wide and multiple communication systems are used cooperatively.

\section{References}

[1] J. Mitola, "The software radio architecture," IEEE Commun. Mag., vol.33, no.5, pp.26-38, May 1995.

[2] K. Araki, "Software defined radio and its applications," Sipec, pp.119-124, 2002.

[3] D. Akos, M. Stockmaster, J.B.Y. Tsui, and J. Caschera, "Direct bandpass sampling of multiple distinct RF signals," IEEE Trans. Commun., vol.47, no.7, pp.983-988, July 1999.

[4] G. Hill, "The benefits of undersampling," Electron. Design, pp.69-79, July 1994.

[5] R.G. Vaughan, N.L. Scott, and D.R. White, "The theory of bandpass sampling," IEEE Trans. Signal Process., vol.39, pp.1973-1984, Sept. 1991.

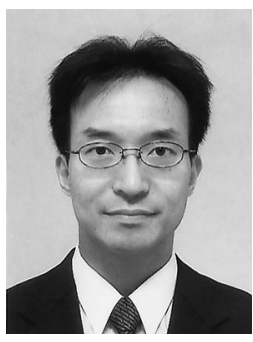

Yoshio Kunisawa received B.E. degree in Electronics and Communications, and M.E. degree in Electrical Engineering from Meiji University, Tokyo, in 1991 and 1993, respectively. Since 1993, he has worked as an electronics engineer in SONY Corporation. During this time, he worked on telecommunication projects, including the Personal Handyphone System (PHS) and the Multimedia Mobile Access Communication system (MMAC). From 1999, he works in KDDI R\&D Laboratories, and researches radio systems such as wireless access system, Ultra WideBand (UWB) and Software Defined Radio (SDR).

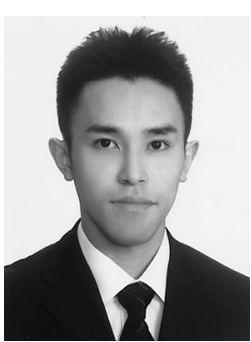

Naohiro Sahara was born in Shizuoka, Japan, on April 15, 1980. He received the B.S and M.S. degrees in electrical, electronic, and communication engineering at Chuo University, Tokyo, Japan, in 2003 and 2005. He now work for KDDI since April 2005.

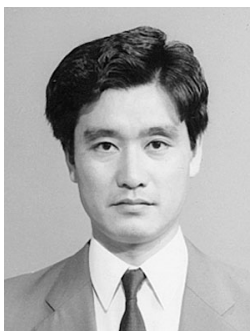

Hiroshi Shirai was born in Aichi, Japan, on March 17, 1958. He received the B.S. and M.S. degrees in electrical engineering from Shizuoka University, Japan, in 1980 and 1992, respectively, and the Ph.D. degree in electrical engineering from Polytechnic University, New York in 1986. He was a Research Fellow, then a Postdoctoral Scientist until March 1987 in Polytechnic University. Since April 1987, he has been with Chuo University, Tokyo, Japan, where he is currently a Professor. He has been serving as a committee member of various societies and international meetings. Recently, he was a Secretary General (1994-1996)of the Engineering Sciences Society of the IEICE, an Associate Editor (1999-2001)of the Journal of the IEICE, and an Editor-in-Chief (2002-2004) of the Transactions of the IEICE A. He is now an Associate Editor of the IEICE Transactions on Electronics. He received the R.W.P. King Best Paper Award from the Antennas and Propagation Society of the IEEE in 1987. His current research interests are wave propagation and diffraction in time harmonic and transient domains. Dr. Shirai is a senior member of the IEEE, and a member of Sigma Xi, the IEE of Japan, the Acoustical Society of Japan (ASJ), and the Acoustical Society of America (ASA).

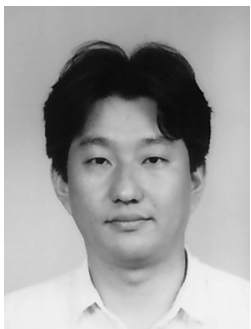

Hisato Iwai received B.S., M.S. and Ph.D degrees from Kyoto University in 1987, 1989 and 2001, respectively. In 1989, he joined KDD R\&D Labs., where he was engaged in research on antennas and propagations in radio communication systems. From 1996 to 1997 he was a visiting scholar at University of California, San Diego (UCSD). Also he was with Telcordia Technologies, NJ, U.S.A. as a visiting researcher from 1999 to 2001. From 2004 to 2005 , he was a research head at ATR Adaptive Communications Research Laboratories. He is currently an Associate Professor at Doshisha University, Kyoto Japan. His current research interests include propagation models in mobile environments, transmission systems in the next generation mobile systems, SDR, wireless adhoc networks and so on. Dr. Iwai is a member of the IEEE. 